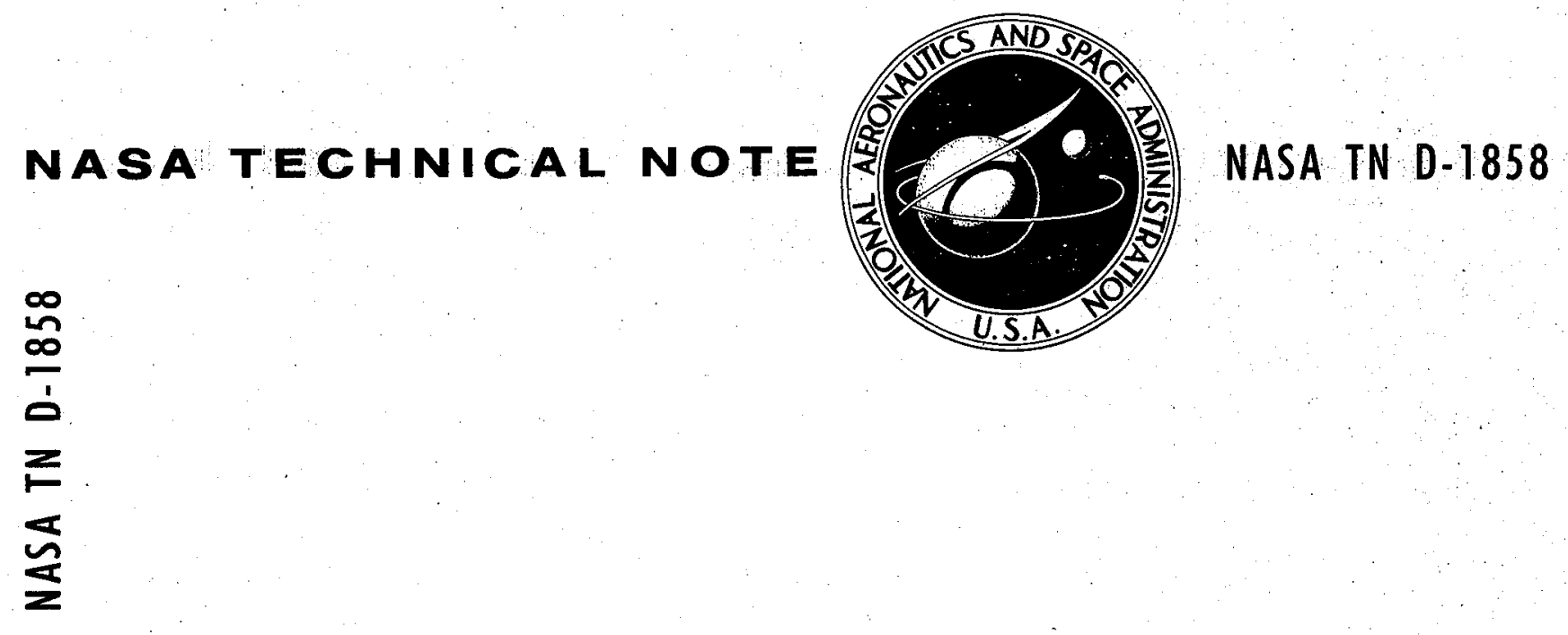

\title{
A CONTRIBUTION TO THE THEORY \\ OF CRITICAL INCLINATION OF \\ CLOSE EARTH SATELLITES
}

by Shinko Aoki;

Goddard Space Flight Center,

Greenbelt, Maryland

NATIONAL AERONAUTICS AND SPACE ADMINISTRATION - WASHINGTON, D. C. - SEPTEMBER 1963 
TECHNICAL NOTE D-1858

A CONTRIBUTION TO THE THEORY OF CRITICAL INCLINATION

OF CLOSE EARTH SATELLITES

By Shinko Aoki

Goddard Space Flight Center

Greenbelt, Maryland 


\title{
A CONTRIBUTION TO THE THEORY OF CRITICAL INCLINATION OF CLOSE EARTH SATELLITES
}

\author{
by \\ Shinko Aoki \\ Goddard Space Flight Center
}

\begin{abstract}
SUMMARY
A higher order theory of the motion of close satellites in the vicinity of the critical inclination is developed for the case of small eccentricity as well as moderate eccentricity. Terms up to the third order of magnitude are included for a potential which is plane symmetrical. It is shown that solutions up to this order can be obtained by using only Jacobian elliptic functions for the case of small eccentricity. However, for moderate eccentricity, elliptic integrals of the second kind or the Jacobian zeta-function are required. To obtain further approximations, elliptic integrals of the third kind are necessary for the case of small eccentricity; but, for the case of moderate eccentricity only the Jacobian elliptic functions and elliptic integrals of the second kind are required. In any case, the solutions are, in general, periodic functions-as is well known from the form of the equations of motion.
\end{abstract}


Page Intentionally Left Blank 
CONTENTS

Summary $\ldots \ldots \ldots \ldots \ldots \ldots \ldots \ldots \ldots \ldots \ldots$

INTRODUCTION. ...................... 1

EQUATIONS OF MOTION .................. 3

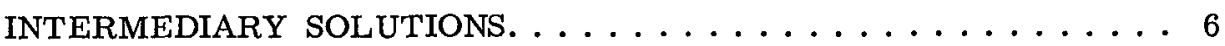

THE CASE OF SMALL ECCENTRICITY............ 11

THE CASE OF MODERATE ECCENTRICITY. . . . . . . . 16

THE GENERAL SCHEME OF THE FURTHER APPROXIMATIONS . . 20

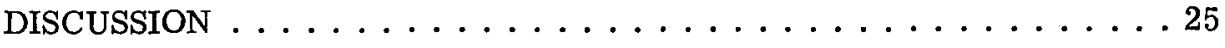

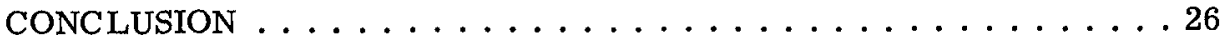

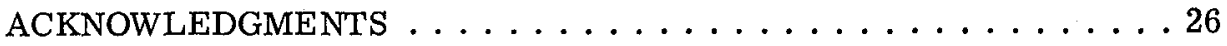

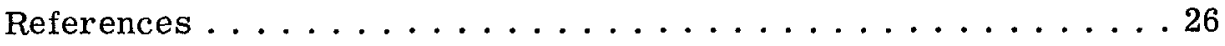

Appendix A-List of Symbols . . . . . . . . . . . . 29 29 


\title{
A CONTRIBUTION TO THE THEORY OF CRITICAL INCLINATION OF CLOSE EARTH SATELLITES*
}

\author{
by \\ Shinko Aoki ${ }^{\dagger}$ \\ Goddard Space Flight Center
}

\section{INTRODUCTION}

Many authors have shown their interest in the motion of close earth satellites near the critical inclination (for example, References 1-5). However, almost all of these authors have considered only the first terms, which are of the order of the square of $J_{2}$ in the equations of motion. However, it was first shown by Izsak (Reference 5) that continuations of the ordinary treatment of the libration to the higher approximations would break down; and, in the case of small eccentricity, some libration of a peculiar kind including the next order of magnitude would occur. The first term, which has $e_{0}^{2}$ as a factor, is so small that the next order term-which would be considered as a higher order of magnitude in the normal case-becomes the same order of magnitude as the preceding term. However, he presented this only for a consideration of the form of the Hamiltonian; he did not try to solve the equations of motion with time as the independent variable.

Here some expressions for these solutions will be presented, not only for the case of small eccentricity, but also for the normal case including terms up to the third order of $\mathrm{J}_{2}$ in the original equations of motion, where $\mathrm{J}_{2}$ is the coefficient of second zonal harmonics of the earth's potential and $\mathrm{J}_{4}$ is assumed to be of the order of the square of $\mathrm{J}_{2}$ and, further, $\mathrm{J}_{6}$ to be of the third order of magnitude. The odd harmonics will be totally neglected. A higher order theory including these terms is under consideration, but must be treated in the future.

The method adopted is to introduce intermediary solutions, of which the argument $\lambda u$ is only slightly different from time (the independent variable) except for a constant factor, and such that by which the amplitude of the libration or the change of states-in other words, a transition from revolution to libration-is fully determined. In the case of small eccentricity, the intermediary solution can be obtained by the following integration:

$$
\frac{d \omega_{1}}{\sqrt{\left(1-\alpha \sin ^{2} \omega_{1}\right)\left(1-\beta \sin ^{2} \omega_{1}\right)}}=\lambda d u
$$

"Published in substantially the same form in Astronom. J. 68(7):355-364, August 1963.

†NAS-NASA Research Associate. 
where $\pi / 2-\omega_{1}$ is the mean value of the argument of perigee and $\lambda$ is constant; this integration can be performed even if only the Jacobian elliptic functions are used, as will be seen in a later section.*

Further approximations, beyond the aforementioned order of magnitude, would require more complicated calculations. Some comments will be made on the subject although explicit formulas are not included. Izsak, after removing the so-called periodic perturbations, wrote the equations of motion:

$$
\begin{aligned}
& \frac{\mathrm{d} \rho}{\mathrm{d} \tau}=\frac{\partial \Phi}{\partial \omega}, \\
& \frac{\mathrm{d} \omega}{\mathrm{d} \tau}=-\frac{\partial \Phi}{\partial \rho},
\end{aligned}
$$

where $\omega$ is the mean value of the argument of perigee; $\rho$ is some function of $\mathrm{G}$, the conjugate variable to $\omega ;$ and

$$
\begin{aligned}
& \Phi=A(\rho)+j B(\rho) \cos ^{2} \omega \\
& A(\rho)=\rho^{2}+\sum_{3} \alpha_{n} \rho^{n} \\
& B(\rho)=\sum_{0} \beta_{n} \rho^{n} \\
& \beta_{0}=e_{0}^{2}[1+o(j)] \\
& \beta_{1}=-2[1+O(j)] \\
& j=-\frac{1}{5}\left(\frac{J_{4}}{J_{2}}+J_{2}\right) p_{0}^{-2}>0 \\
& \tau=\frac{3}{4} \frac{J_{2} n_{0}}{\zeta p_{0}^{2}}\left(t-t_{0}\right)
\end{aligned}
$$

Since $\tau$ has a factor of $J_{2}$, the terms of the order of $\mathrm{j}$ in $\Phi$ correspond to those of the order of $\mathrm{J}_{2}^{2}$ or $\mathrm{J}_{4}$ in the original equations of motion.

In this paper we have started with a Hamiltonian similar to the one used by Izsak but have added some additional terms in order to more easily consider the higher order terms; although this explicit evaluation, from the coefficients of zonal harmonics of the earth's potential and from the integration

*A list of symbols is given in Appendix A. 
constants, is not given. We are concerned with the solutions of the equations of motion which contain the Hamiltonian up to the order of $\mathrm{j}^{2}$ :

$$
\Phi=\mathrm{A}(\rho)+\mathrm{j} \mathrm{B}(\rho) \cos ^{2} \omega+\mathrm{j}^{2} \mathrm{C}(\rho) \cos ^{4} \omega
$$

although some comments will be made on obtaining higher order approximations.

\section{EQUATIONS OF MOTION}

We shall now consider the following equations:

$$
\begin{aligned}
& \frac{\mathrm{d} \rho}{\mathrm{d} \tau}=\frac{\partial \Phi}{\partial \omega}, \\
& \frac{\mathrm{d} \omega}{\mathrm{d} \tau}=-\frac{\partial \Phi}{\partial \rho},
\end{aligned}
$$

where

$$
\begin{aligned}
\Phi & =\mathbf{A}(\rho)+j B(\rho) \cos ^{2} \omega+\mathbf{j}^{2} \mathrm{C}(\rho) \cos ^{4} \omega, \\
\mathbf{A}(\rho) & =\sum_{\mathbf{n}=2}^{\infty} \alpha_{\mathrm{n}} \rho^{\mathrm{n}}, \\
\mathbf{B}(\rho) & =\sum_{0}^{\infty} \beta_{\mathrm{n}} \rho^{\mathrm{n}}, \\
\mathrm{C}(\rho) & =\sum_{0}^{\infty} \gamma_{\mathrm{n}} \rho^{\mathrm{n}},
\end{aligned}
$$

and $\alpha_{n}, \beta_{n}, \gamma_{n}$ are constants which satisfy $\alpha_{2}>0, \beta_{0}>0$.

In order to remove the first degree term of $\rho$ in the Hamiltonian function, we change the variable $\rho$ to $x=\rho-p\left(\cos ^{2} \omega\right)$ such that

$$
\left(\frac{\partial \Phi}{\partial \chi}\right)_{X=0}=\left(\frac{\partial \Phi}{\partial \rho}\right)_{\rho=\mathbf{p}}:=0
$$

If we take the terms up to $j^{2}$ in Equation 2, we have

$$
\mathrm{j} \beta_{1} \cos ^{2} \omega+\mathrm{j}^{2} \gamma_{1} \cos ^{4} \omega+2\left(\alpha_{2}+\mathrm{j} \beta_{2} \cos ^{2} \omega\right) \mathrm{p}+3 \alpha_{3} \mathrm{p}^{2}=0
$$


from which

$$
P=-\frac{1}{2} j \frac{\beta_{1}}{a_{2}} \cos ^{2} \omega+j^{2}\left(-\frac{1}{2} \frac{\gamma_{1}}{a_{2}}+\frac{1}{2} \frac{\beta_{1} \beta_{2}}{\alpha_{2}^{2}}-\frac{3}{8} \frac{a_{3} \beta_{1}^{2}}{a_{2}^{3}}\right) \cos ^{4} \omega
$$

Thus, the equations,

$$
\begin{aligned}
& \frac{\mathrm{d} \chi}{\mathrm{d} \tau}=\frac{\partial \Psi}{\partial \omega}, \\
& \frac{\mathrm{d} \omega}{\mathrm{d} \tau}=-\frac{\partial \Psi}{\partial \chi},
\end{aligned}
$$

are obtained with

$$
\begin{aligned}
\Psi(\chi, \omega) & =\Phi(p+\chi, \omega) \\
& =a_{0}+a_{2} \chi^{2}+a_{3} \chi^{3}+a_{4} \chi^{4}+\cdots,
\end{aligned}
$$

where

$$
\begin{aligned}
& a_{0}=j \beta_{0} \cos ^{2} \omega+j^{2}\left(-\frac{1}{4} \beta_{1}^{2} a_{2}^{-1}+\gamma_{0}\right) \cos ^{4} \omega+\cdots, \\
& a_{2}=a_{2}+j\left(\beta_{2}-\frac{3}{2} a_{3} \beta_{1} a_{2}^{-1}\right) \cos ^{2} \omega+\cdots, \\
& a_{3}=a_{3}+\cdots \\
& a_{4}=a_{4}+\cdots .
\end{aligned}
$$

We have considered terms up to the order of $j^{2}$ in $\Psi$, assuming $x$ is the order of $j^{1 / 2}$. We know that

$$
\Psi=\mathrm{j} \Gamma
$$

where $\Gamma$ is constant, is an integral of Equation 4. Neglecting higher order terms, we can easily obtain an expression for $\chi$ from Equation 6:

$$
x=x_{1}-\frac{1}{2} a_{3} a_{2}^{-1} x_{1}^{2}+\left(-\frac{1}{2} a_{4} a_{2}^{-1}+\frac{5}{8} a_{3}^{2} a_{2}^{-2}\right) x_{1}^{3},
$$


where

$$
\begin{aligned}
x_{1}^{2}=\left(j \Gamma-a_{0}\right) a_{2}^{-1} ; \\
=\alpha_{2}^{-1} j\left[\Gamma-\beta_{0} \cos ^{2} \omega+j\left(\frac{1}{4} \beta_{1}^{2} \alpha_{2}^{-1}-\gamma_{0}\right) \cos ^{4} \omega\right. \\
\\
\left.\quad+j\left(\Gamma-\beta_{0} \cos ^{2} \omega\right)\left(-\beta_{2} \alpha_{2}^{-1}+\frac{3}{2} \alpha_{3} \beta_{1} \alpha_{2}^{-2}\right) \cos ^{2} \omega\right] .
\end{aligned}
$$

Rewriting $x_{1}^{2}$ in the form

$$
\chi_{1}^{2}=\mu\left(1+P_{1} \cos ^{2} \omega+Q_{1} \cos ^{4} \omega\right),
$$

where

$$
\begin{aligned}
\mu & =\Gamma \alpha_{2}^{-1} \mathbf{j}, \\
P_{1} & =\Gamma^{-1}\left[-\beta_{0}+\mathrm{j} \Gamma\left(-\beta_{2} \alpha^{-1}+\frac{3}{2} \alpha_{3} \beta_{1} \alpha_{2}^{-2}\right)\right], \\
Q_{1} & =\mathrm{j} \Gamma^{-1}\left(\frac{1}{4} \beta_{1}^{2} \alpha_{2}^{-1}-\gamma_{0}+\beta_{0} \beta_{2} \alpha_{2}^{-1}-\frac{3}{2} \alpha_{3} \beta_{0} \beta_{1} \alpha_{2}^{-2}\right),
\end{aligned}
$$

and putting $\omega_{1}=\pi / 2-\omega$, we have

$$
\begin{aligned}
\frac{d \omega_{1}}{2 a_{2} \chi+3 a_{3} \chi^{2}+4 a_{4} \chi^{3}} & =\frac{d \omega_{1}}{2 \alpha_{2} \mu^{1 / 2} \chi_{0}\left[1+\alpha_{3} \alpha_{2}^{-1} \mu^{1 / 2} \chi_{0}+\left(\frac{3}{2} \alpha_{4} \alpha_{2}^{-1}-\frac{7}{8} \alpha_{3}^{2} a_{2}^{-2}\right) \mu \chi_{0}^{2}\right]} \\
& =d \tau,
\end{aligned}
$$

where

$$
\left.\begin{array}{rl}
x_{0} & =a_{2} a_{2}^{-1} \mu^{-1 / 2} \chi_{1} \\
& =\sqrt{1+P \sin ^{2} \omega_{1}+Q \sin ^{4} \omega_{1}} \\
P & =-\beta_{0} \Gamma^{-1}+j\left(\beta_{2} a_{2}^{-1}-\frac{3}{2} \alpha_{3} \beta_{1} a_{2}^{-2}\right) \\
Q & =j \Gamma^{-1}\left(\frac{1}{4} \beta_{1}^{2} \alpha_{2}^{-1}-\gamma_{0}-\beta_{0} \beta_{2} \alpha_{2}^{-1}+\frac{3}{2} \alpha_{3} \beta_{0} \beta_{1} \alpha_{2}^{-2}\right) .
\end{array}\right\}
$$

5 


\section{INTERMEDIARY SOLUTIONS}

From the denominator on the right side of Equation 8 we can see that it will be zero if, and only if, $\chi_{0}=0$, provided $\mu=\mathrm{O}(\mathrm{j} \Gamma)$ is small. This means that the essential character of the trajectory is determined entirely by $\chi_{0}$. In the case when $\omega_{1}$ is real-which includes, in general, $j^{2}$ terms of the equation of motion. Thus, $\chi_{0}$ discriminates among three kinds of motion: libration, revolution, and the critical case.

Therefore, we first must consider

$$
\begin{aligned}
\frac{\mathrm{d} \omega_{1}}{\sqrt{1+\mathrm{P} \sin ^{2} \omega_{1}+\mathrm{Q} \sin ^{4} \omega_{1}}} & =\frac{\mathrm{d} \omega_{1}}{\sqrt{\left(1-\alpha \sin ^{2} \omega_{1}\right)\left(1-\beta \sin \omega_{1}\right)}} \\
& =\mathrm{du} .
\end{aligned}
$$

Putting $\sin ^{2} \omega_{1}=x$, we have

$$
d u=\frac{d x}{2 \sqrt{(1-x) \times\left(1+P x+Q x^{2}\right)}}
$$

and, putting $y=x^{-1}$, we have

$$
d u=\frac{-d y}{\sqrt{4(y-1)\left(y^{2}+P y+Q\right)}}=\frac{-d y}{\sqrt{4(y-1)(y-\alpha)(y-\beta)}},
$$

or

$$
d u=\frac{-d z}{\sqrt{4\left(z-e_{f}\right)\left(z-e_{g}\right)\left(z-e_{h}\right)}},
$$

where

$$
z=y+\frac{P-1}{3}
$$

and

$$
\left(e_{f}, e_{g}, e_{h}\right)=\left(\frac{P}{3}+\frac{2}{3}, \alpha+\frac{P-1}{3}, \beta+\frac{P-1}{3}\right) .
$$

Thus, we have

$$
z=\wp\left(u-u_{0}\right),
$$


where $\wp \mathrm{u}$ is the Weierstrassian elliptic function of the second order. We assume that the integration constant is such that

$$
u_{0}=0 \text {, }
$$

which corresponds to the initial condition of $\omega_{1}=0$ when $u=0$, as will be seen later. Then we have

$$
\begin{aligned}
\sin ^{2} \omega_{1} & =\left(\wp u-\frac{P-1}{3}\right)^{-1}=\left(\wp u-e_{f}+1\right)^{-1} \\
& =\left(1+f^{2} u\right)^{-1},
\end{aligned}
$$

or

$$
\cot \omega_{1}= \pm \mathrm{fju}
$$

and

$$
\sqrt{1+P \sin ^{2} \omega_{1}+Q \sin ^{4} \omega_{1}}=g j u \cdot h j u\left(1+f j^{2} u\right)^{-1}
$$

In the above expressions, $\mathrm{f} j \mathrm{j}$ is an associated primitive elliptic function of the first order (Reference 6), whose poles are $u=0$ and $2 \omega_{\mathrm{g}}$ and whose zeros are $u=\omega_{\mathrm{f}}$ and $\omega_{\mathrm{f}}+2 \omega_{\mathrm{g}}$ within a parallelogram $\left(2 \omega_{f}, 4 \omega_{g}\right)$, where we have

$$
\omega_{p}=\int_{e_{p}}^{\infty} \frac{d z}{\sqrt{4\left(z-e_{f}\right)\left(z-e_{g}\right)\left(z-e_{h}\right)}},
$$

with $p=f, g, h$ and $\omega_{f}+\omega_{g}+\omega_{h}=0$. The other $g j u$ and $h j u$ are given by permutations of $(f, g, h)$.

However, this expression is so general that, at first glance, we cannot see how the function behaves for real values of $u$, which is the only case of interest. Therefore, in order to clarify its behavior, we shall divide the problem into several cases.

(i) a, $\beta$ real: Libration and critical cases

(a) $1 \leq \beta<a \leq \mathrm{y}$, libration within $-\alpha^{-1 / 2} \leq \sin \omega_{1} \leq \alpha^{-1 / 2}$

Since

$$
\wp \mathrm{u}=1+\frac{\mathrm{p}-1}{3}+\frac{a-1}{\mathrm{sn}^{2}(\lambda \mathrm{u}, \mathrm{k})}
$$


where $\lambda^{2}=\alpha-1$ and $k^{2}=(\beta-1) /(\alpha-1)$, we have

$$
\sin ^{2} \omega_{1}=\frac{\operatorname{sn}^{2}(\lambda u, k)}{\alpha-\operatorname{cn}^{2}(\lambda u, k)}
$$

and

$$
x_{0}=\sqrt{1+P \sin ^{2} \omega_{1}+Q \sin ^{4} \omega_{1}}=\frac{(\alpha-1) \operatorname{cn} \lambda u \operatorname{dn} \lambda u}{\alpha-\operatorname{cn}^{2}(\lambda u, k)} .
$$

The case $1 \leq y \leq \beta \leq \alpha$ does not correspond to any real case in the theory of the critical inclination of close artificial satellites (Reference 5); therefore, we omit this case here, although similar expressions are obtainable if we substitute $\sin \omega=\cos \omega_{1}$ instead of $\sin \omega_{1}$ in Equation 1 .

(b) $\beta<1<\alpha \leq y$, libration within $-\alpha^{-1 / 2} \leq \sin \omega_{1} \leq \alpha^{-1 / 2}$

Since

$$
\text { øu }=\beta+\frac{P-1}{3}+\frac{a-\beta}{\operatorname{sn}^{2}(\lambda u, k)} \text {, }
$$

where $\lambda^{2}=\alpha-\beta$ and $\mathrm{k}^{2}=(1-\beta) /(\alpha-\beta)$, we have

$$
\sin ^{2} \omega_{1}=\frac{\operatorname{sn}^{2}(\lambda \mathrm{u}, \mathrm{k})}{\alpha-\beta \mathrm{cn}^{2}(\lambda \mathrm{u}, \mathrm{k})}
$$

and

$$
\begin{aligned}
& x_{0}=\frac{(\alpha-\beta) \mathrm{cn}(\lambda \mathrm{u}, \mathrm{k})}{\alpha-\beta \mathrm{cn}^{2}(\lambda \mathrm{u}, \mathrm{k})} . \\
& \text { (c) } \beta \leq \alpha<1 \leq \mathrm{y}, \text { revolution }
\end{aligned}
$$

Since

$$
\wp \mathrm{u}=\beta+\frac{\mathrm{P}-1}{3}+\frac{1-\beta}{\operatorname{sn}^{2}(\lambda \mathrm{u}, \mathrm{k})},
$$

where $\lambda^{2}=1-\beta$ and $k^{2}=(\alpha-\beta) /(1-\beta)$, we have

$$
\sin ^{2} \omega_{1}=\frac{\operatorname{sn}^{2}(\lambda \mathrm{u}, \mathrm{k})}{1-\beta \mathrm{cn}^{2}(\lambda \mathrm{u}, \mathrm{k})}
$$


and

$$
\begin{aligned}
& x_{0}=\frac{(1-\beta) \mathrm{dn}(\lambda \mathrm{u}, \mathrm{k})}{1-\beta \mathrm{cn}^{2}(\lambda \mathrm{u}, \mathrm{k})} . \\
& \text { (a') } 1<\beta=\alpha \leq \mathrm{y}, \text { critical }
\end{aligned}
$$

Since $\operatorname{sn} \lambda u \rightarrow \tanh \lambda u \quad$ when $k \rightarrow 1$, we have

$$
\sin ^{2} \omega_{1}=\frac{\sinh ^{2} \lambda_{u}}{\alpha \cosh ^{2} \lambda u-1}
$$

where $\lambda^{2}=\alpha-1$ and

$$
\begin{gathered}
x_{0}=\frac{\alpha-1}{\alpha \cosh ^{2} \lambda u-1} . \\
\text { (b') } \beta<1=a \leq y, \text { critical }
\end{gathered}
$$

From either case b or c, when $\mathrm{k} \rightarrow 1$, we have

$$
\sin ^{2} \omega_{1}=\frac{\sinh ^{2} \lambda u}{\cosh ^{2} \lambda u-\beta}
$$

and

$$
\chi_{0}=\frac{(1-\beta) \cosh \lambda_{u}}{\cosh ^{2} \lambda u-\beta},
$$

where $\lambda^{2}=1-\beta$.

(ii) a, B imaginary: Revolution

Here the direct transformation from $\wp$-functions to Jacobian elliptic functions does not give a real function. Therefore, another type of transformation is used, as is described by Whittaker and Watson (Reference 7, p. 513), since $P$ and Q are real.

If we apply the transformation

$$
y=C \frac{x-B}{x-A}
$$

where

$$
A=\frac{-1-\sqrt{1+P+Q}}{P+Q}
$$




$$
\begin{aligned}
& B=\frac{-1+\sqrt{1+P+Q}}{P+Q}, \\
& C=\frac{-2-P-Q-2 \sqrt{1+P+Q}}{P+Q},
\end{aligned}
$$

to Equation 11, we then have

$$
\begin{aligned}
\mathrm{du} & =\frac{\mathrm{d} \omega_{1}}{\sqrt{1+P \sin ^{2} \omega_{1}+Q \sin ^{4} \omega_{1}}} \\
& =\frac{d y}{2 \lambda \sqrt{\left(1-y^{2}\right)\left(k^{\prime 2}+k^{2} y^{2}\right)}}
\end{aligned}
$$

where

$$
\begin{gathered}
\lambda^{2}=\sqrt{1+\mathrm{P}+\mathrm{Q}}=\sqrt{(1-\alpha)(1-\beta)}, \\
\mathrm{k}^{2}=\frac{-\mathrm{P}-2+2 \sqrt{1+\mathrm{P}+\mathrm{Q}}}{4 \sqrt{1+\mathrm{P}+\mathrm{Q}}}=\frac{\alpha+\beta-2}{4 \sqrt{(1-\alpha)(1-\beta)}}+\frac{1}{2} .
\end{gathered}
$$

Accordingly, we obtain

$$
\mathrm{y}=\operatorname{cn}\left[2 \lambda\left(\mathrm{u}-\mathrm{u}_{0}\right), \mathrm{k}\right]
$$

It should be noted that the form of $y$ in Equation 14 yields a real function, when $\lambda^{2} \geq 0$ and $1 \geq k^{2} \geq 0$. This is made possible by choosing an appropriate sign of the above root function (which is, of course, a double-valued function) in such a way that

$$
\sqrt{1+P+Q} \geq 0
$$

this is possible because $P$ and $Q$ are real and satisfy the inequalities

$$
\begin{gathered}
1+P+Q=\left(1+\frac{P}{2}\right)^{2}-\frac{1}{4}\left(P^{2}-4 Q\right) \geq 0, \\
(P+2)^{2} \leq 4(1+P+Q),
\end{gathered}
$$

since

$$
\Delta=P^{2}-4 Q \leq 0 .
$$


Table 1

List of Solutions for $d \omega_{1}=\chi_{0} d u, \chi_{0}^{2}=(1-\alpha x)(1-\beta x), x=\sin ^{2} \omega_{1}=\cos ^{2} \omega$.

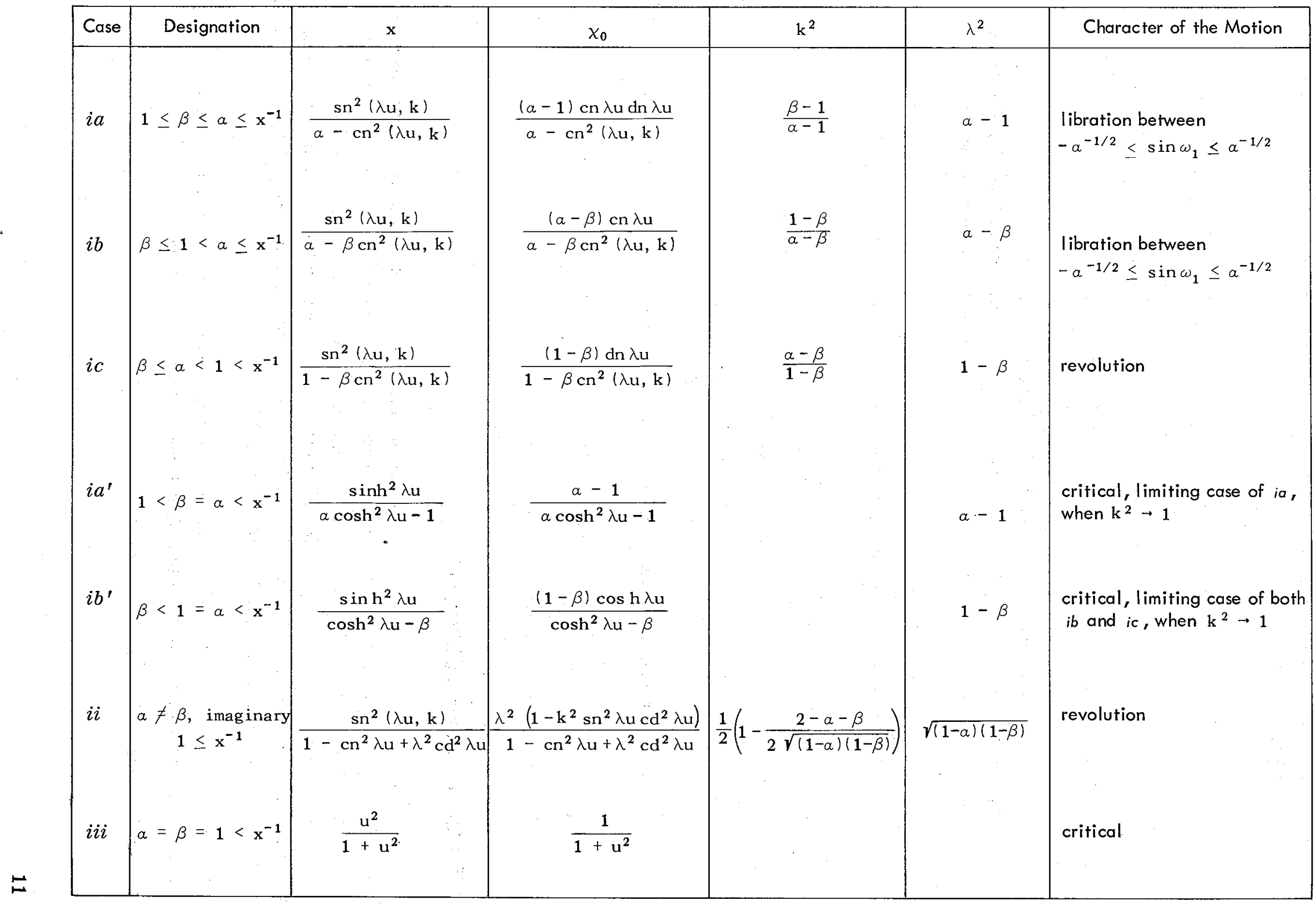


Choosing the integration constant $\mathrm{u}_{0}=0$, and using the addition theorem, we have the following expressions:

$$
x=\sin ^{2} \omega_{1}=\frac{\operatorname{sn}^{2}(\lambda u, k)}{1-\operatorname{cn}^{2} \lambda u+\lambda^{2} \operatorname{cd}^{2} \lambda u}
$$

and

$$
x_{0}=\frac{\lambda^{2}\left(1-\mathrm{k}^{2} \mathrm{sn}^{2} \lambda \mathrm{u} \mathrm{cd} \mathrm{d}^{2} \lambda \mathrm{u}\right)}{1-\mathrm{cn}^{2} \lambda \mathrm{u}+\lambda^{2} \mathrm{~cd}^{2} \lambda \mathrm{u}}
$$

Consider the critical case when the descriminant $\Delta$ goes to zero. If $-\mathrm{P}=\alpha+\beta>2$, then $\mathrm{k}^{2}$ approaches to unity; and the above expressions, of course, tend to Case $i{ }^{\prime}$. On the other hand, if $-\mathrm{P}=\alpha+\beta<2$, then $\mathrm{k}^{2} \rightarrow 0$; and this case tends to be a special case of Case $i c$.

(iii) $\alpha=\beta=1$, critical.

In this case Equation 10 may be expressed directly as

$$
\frac{d \omega}{\sin ^{2} \omega}=-d u
$$

from which we obtain $\sin \omega=1 / \sqrt{u^{2}+1}$, or

$$
\sin \omega_{1}=\frac{u}{\sqrt{u^{2}+1}},
$$

where the integration constant is chosen such that $\omega_{1}=0$ when $u=0$. Consequently,

$$
x_{0}=\cos ^{2} \omega_{1}=\frac{1}{u^{2}+1} .
$$

Table 1 summarizes the data from the three cases.

\section{THE CASE OF SMALL ECCENTRICITY}

If $\beta_{0}\left(\approx e_{0}^{2}\right)$ and $\Gamma$ are of the same order of magnitude as $j$, then the argument given for the equations of motion becomes somewhat simpler because $\chi$ is of the order of $\mathrm{j}$ so that the terms $a_{3} \chi^{3}$ and $\alpha_{4} \chi^{4}$ in Equations 5 are negligible provided we include terms up to the second order in these equations. Moreover, in the expression $\mu_{\chi_{0}}{ }^{2}$ it is sufficient to consider only terms up to the second order. In the case of small eccentricity near the critical inclination, $\gamma_{0}$ itself (Equations 5 ) would be of the order of $j^{2}$, which is also negligible (Reference 8 ).

Thus, in this case, the intermediary solutions described in a previous section would give sufficient accuracy provided that we take Equations 1 to the second order.

Now putting $\beta_{0}=\mathrm{j} \beta_{0}^{\prime}, \Gamma^{\prime}=\mathrm{j} \Gamma^{\prime}$ we have

$$
P=-\beta_{0}^{\prime} \Gamma^{\prime-1}
$$




$$
\begin{gathered}
Q=\frac{1}{4} \Gamma^{\prime-1} \beta_{1}^{2} \alpha_{2}^{-1}, \\
\mu=j^{2} \Gamma^{\prime} \alpha_{2}^{-1} ;
\end{gathered}
$$

from which we obtain

$$
(\alpha, \beta)=\frac{1}{2} \beta_{0}^{\prime} \Gamma^{\prime-1}\left(1 \pm \sqrt{1-\Gamma^{\prime} \beta_{1}^{2} \beta_{0}^{\prime-2} a_{2}^{-1}}\right) .
$$

From Equation 8 we have

$$
u=2 \alpha_{2} \sqrt{\mu} \tau
$$

where

$$
\mu=j^{2} \Gamma^{\prime} a_{2}^{-1} .
$$

If $\Gamma^{\prime}>\beta_{0}^{\prime 2} \alpha_{2} \beta_{1}^{-2}, \alpha$ and $\beta$ become imaginary so that this case corresponds to the Case $i i$.

On the other hand, $\Gamma^{\prime} \leq \beta_{0}^{\prime 2} \alpha_{2} \beta_{1}^{-2}$ corresponds to Case $i$ or Case $i$ ii . The subdivision within Case $i$ can be obtained easily

if

$$
\beta_{0}^{\prime}-\frac{\beta_{1}^{2}}{4 a_{2}}<\Gamma^{\prime}<\frac{\beta_{0}^{\prime}}{2}, \text { then }
$$

Case $i a$;

if

$$
0<\Gamma^{\prime}<\beta_{0}^{\prime}-\frac{\beta_{1}^{2}}{4 a_{2}} \text {, then }
$$

Case ib;

and if

$$
\Gamma^{\prime}>\beta_{0}^{\prime}-\frac{\beta_{1}^{2}}{4 \alpha_{2}} \text { and } \frac{\beta_{0}^{\prime}}{2} \text {, then }
$$

Case $i c$.

Rewriting the above and combining it with the real/imaginary criterion results in the following scheme:

Case A

$$
\beta_{0}^{\prime}>\frac{\beta_{1}^{2}}{2 \alpha_{2}} \text { or } \frac{\beta_{0}^{\prime}}{2}<\beta_{0}^{\prime}-\frac{\beta_{1}^{2}}{4 \alpha_{2}}<\frac{\beta_{0}^{\prime 2} \alpha_{2}}{\beta_{1}^{2}}
$$


Case B

if

$$
\beta_{0}^{\prime}<\frac{\beta_{1}^{2}}{2 a_{2}} \text { or } \beta_{0}^{\prime}-\frac{\beta_{1}^{2}}{4 \alpha_{2}}<\frac{\beta_{0}^{\prime 2} \alpha_{2}}{\beta_{1}^{2}}<\frac{\beta_{0}^{\prime}}{2}
$$

if

$$
0<\Gamma^{\prime}<\beta_{0}^{\prime}-\frac{\beta_{1}^{2}}{4 a_{2}}, \text { then }
$$

Case $i b$;

if

$$
\beta_{0}^{\prime}-\frac{\beta_{1}^{2}}{4 \alpha_{2}}<\Gamma^{\prime}<\frac{\beta_{0}{ }^{2} \alpha_{2}}{\beta_{1}^{2}}, \text { then }
$$

Case $i a$;

if

$$
\frac{\beta_{0}^{\prime 2} \alpha_{2}}{\beta_{1}^{2}}<\Gamma^{\prime}, \text { then }
$$

Case $i$.

The critical cases are easily obtained by comparing the above scheme with Table 1 . In order to be able to distinguish between the different cases, a diagram is drawn with the parameters expressed by $\Gamma^{\prime}$ and $\beta_{0}^{\prime}$ in units of $\beta_{1}^{2} / 4 \alpha_{2}$ (Figure 1 ).

Now, the period of $\sin \omega_{1}$ in terms of $\tau$ is given by

$$
T=\frac{4 \mathrm{~K}}{2 \alpha_{2}^{1 / 2} \mathrm{j} \Gamma^{\prime 1 / 2} \lambda}
$$

where the complete elliptic integral of the first kind,

$$
\mathrm{K}=\int_{0}^{\pi / 2} \frac{\mathrm{d} \omega_{1}}{\sqrt{1-\mathrm{k}^{2} \sin ^{2} \omega_{1}}}=\frac{1}{2} \pi \mathrm{F}\left(\frac{1}{2}, \frac{1}{2}, 1, \mathrm{k}^{2}\right) .
$$

Accordingly, if in Figure 1 we take a point which is moving around the critical point iii in a counterclockwise direction, we will find the change of the period and of the character of the motion as given in Table 2. 
In any case the solutions of Equations 1 up to the order considered here, are given in case $i a$ by using Equations 3, 7, 9, 12 and 13;

$$
\cos ^{2} \omega=\sin ^{2} \omega_{1}=\frac{\operatorname{sn}^{2} \lambda u}{a-\operatorname{cn}^{2} \lambda u}
$$

$\rho=p+\chi=p+\mu^{1 / 2} \chi_{0}$

$$
\begin{aligned}
= & -\frac{1}{2} \mathrm{j} \frac{\beta_{1}}{a_{2}} \frac{\mathrm{sn}^{2} \lambda \mathrm{u}}{\alpha-\mathrm{cn}^{2} \lambda \mathrm{u}} \\
& +\mathrm{j} \sqrt{\frac{\Gamma^{\prime}}{a_{2}}} \frac{(\alpha-1) \operatorname{cn} \lambda \mathrm{udn} \lambda \mathrm{u}}{\alpha-\operatorname{cn}^{2} \lambda \mathrm{u}},
\end{aligned}
$$

where

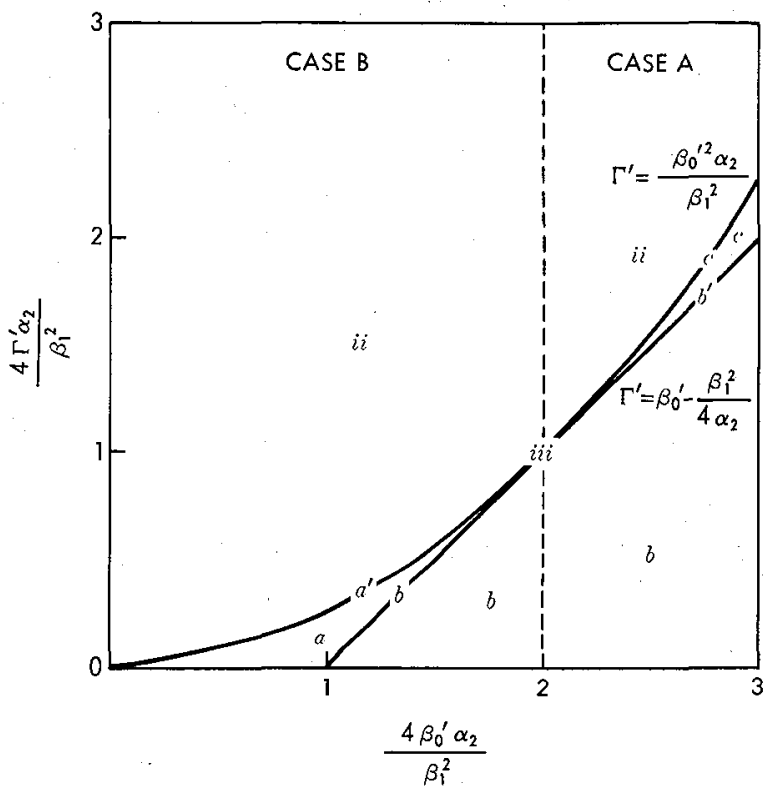

Figure 1-Criterion for several cases, expressed by parameters $\Gamma^{\prime}$ and $\beta_{0}^{\prime}$ in units of $(1 / 4)\left(\beta_{1}{ }^{2} \alpha_{2}{ }^{-1}\right)$ The respective cases are described in Table 1.

$$
\begin{aligned}
\alpha & =\frac{1}{2} \beta_{0}^{\prime} \Gamma^{\prime-1}\left(1+\sqrt{1-\Gamma^{\prime} \beta_{1}^{2} \beta_{0}^{1-2} \alpha_{2}^{-1}}\right), \\
\mathrm{k}^{2} & =\frac{\beta-1}{\alpha-1}, \quad \lambda \mathrm{u}=2 \sqrt{\alpha-1} a_{2}^{1 / 2} \Gamma^{\prime 1 / 2} \mathrm{j} \tau .
\end{aligned}
$$

For the other cases, we can easily obtain the results from Table 1 in a similar manner.

\section{Table 2}

Behavior of the Period and the Character of the Motion.

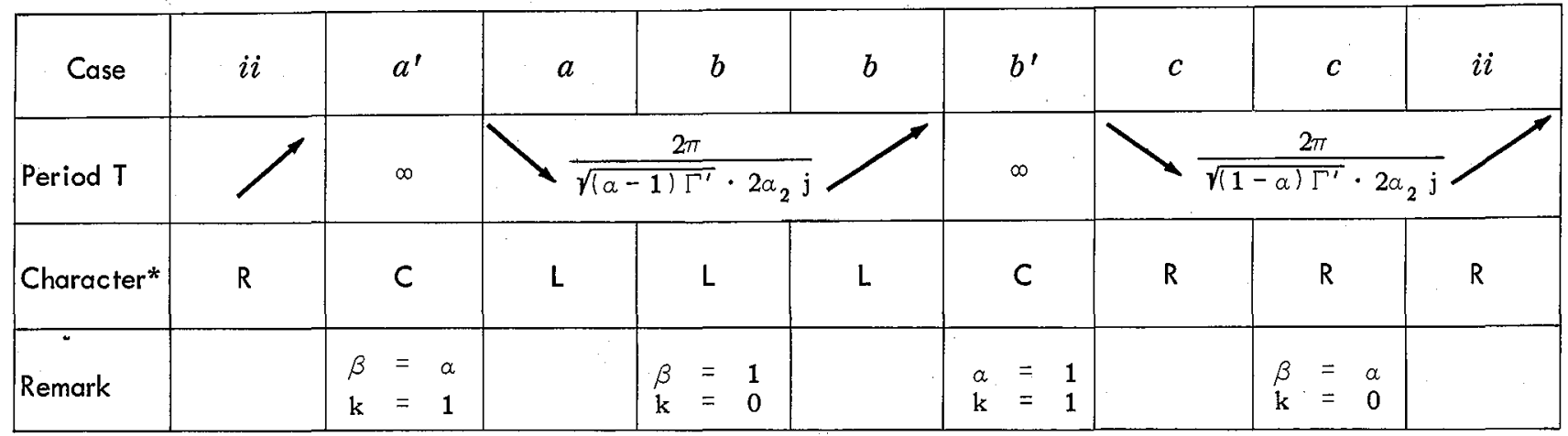

*The abbreviations are as follows: Revolution, Critical, and Libration. 


\section{THE CASE OF MODERATE ECCENTRICITY}

In this case, we expand $\alpha$ and $\beta$ into power series in $j$. From the expressions of Equation 9 it folfows, by neglecting high order terms, that

$$
\begin{aligned}
& \alpha=\frac{\beta_{0}}{\Gamma}-j\left(\frac{1}{4} \frac{\beta_{1}^{2}}{\alpha_{2} \beta_{0}}-\frac{\gamma_{0}}{\beta_{0}}\right), \\
& \beta=j\left(\frac{1}{4} \frac{\beta_{1}^{2}}{\alpha_{2} \beta_{0}}-\frac{\gamma_{0}}{\beta_{0}}-\frac{\beta_{2}}{\alpha_{2}}+\frac{3}{2} \frac{\alpha_{3} \beta_{1}}{\alpha_{2}^{2}}\right) .
\end{aligned}
$$

Here, except for the critical case, we have only one of two cases: $i b$ or $i c$

\section{Case ib: Libration}

From Equations 8 and 10 we obtain

$$
\frac{\mathrm{du}}{1+\frac{a_{3}}{a_{2}} \mu^{1 / 2} \chi_{0}+\left(\frac{3}{2} \frac{a_{4}}{a_{2}}-\frac{7}{8} \frac{a_{3}^{2}}{a_{2}^{2}}\right) \mu \chi_{0}^{2}}=2 \alpha_{2} \mu^{1 / 2} \mathrm{~d} \tau,
$$

from which, expanding it into a power series in $\mu^{1 / 2}$, we obtain

$$
\mathrm{du}\left[1-\frac{a_{3}}{\alpha_{2}} \mu^{1 / 2} \chi_{0}+\left(-\frac{3}{2} \frac{a_{4}}{\alpha_{2}}+\frac{15}{8} \frac{a_{3}^{2}}{a_{2}^{2}}\right) \mu \chi_{0}^{2}\right]=2 \alpha_{2} \mu^{1 / 2} \mathrm{~d} \tau,
$$

up to the order $\mu=O(j)$, which corresponds to $j^{2}$ in Equation 1. We also have, from Equations 7 and 8,

$$
x=\frac{a_{2}}{a_{2}} \mu^{1 / 2} \chi_{0}\left[1-\frac{1}{2} \frac{\alpha_{3}}{\alpha_{2}} \mu^{1 / 2} \chi_{0}+\left(-\frac{1}{2} \frac{\alpha_{4}}{\alpha_{2}}+\frac{5}{8} \frac{a_{3}^{2}}{\alpha_{2}^{2}}\right) \mu \chi_{0}^{2}\right]
$$

Now, putting

$$
\begin{aligned}
& \sigma_{1}=-\frac{\alpha_{3}}{\alpha_{2}}, \\
& \sigma_{2}=-\frac{3}{2} \frac{\alpha_{4}}{\alpha_{2}}+\frac{15}{8} \frac{\alpha_{3}^{2}}{\alpha_{2}^{2}},
\end{aligned}
$$

into Equation 18 and using $\chi_{0}=c n \lambda u$ to the required order, we have

$$
\begin{aligned}
\mathrm{du}\left(1+\sigma_{1} \mu^{1 / 2} \operatorname{cn} \lambda \mathrm{u}+\sigma_{2} \mu \mathrm{cn}^{2} \lambda \mathrm{u}\right) & =2 \alpha_{2} \mu^{1 / 2} \mathrm{~d} \tau \\
& \equiv \mathrm{du}_{1} .
\end{aligned}
$$


It is known that (Reference 9, pp. 58 and 62)

$$
\begin{aligned}
& \int_{0}^{v} \mathrm{cnvdv}=\mathrm{k}^{-1} \sin (\mathrm{k} \operatorname{sn} \mathrm{v}), \quad 0 \leq \mathrm{k}^{2} \leq 1, \\
& \int_{0}^{v} \operatorname{cnv} v^{2} d v=k^{-2}\left[E(v)-k^{\prime 2} v\right]
\end{aligned}
$$

where $E(v)$ is the elliptic integral of the second kind such as

$$
E(v)=\int_{0}^{v} d n^{2} v d v
$$

Accordingly,

$$
\begin{aligned}
\lambda \mathrm{u}_{1}=\lambda\left(1+\sigma_{2} \mu \mathrm{s}\right) \mathrm{u}+\sigma_{1} \mu^{1 / 2} \mathrm{k}^{-1} \sin ^{-1}(\mathrm{k} \mathrm{sn} \lambda \mathrm{u})+\mathrm{k}^{-2} \sigma_{2} \mu \mathrm{Z}(\lambda \mathrm{u}), \\
Z(\mathrm{v})=\mathrm{E}(\mathrm{v})-\mathrm{EK}^{-1} \mathrm{v}, \\
\mathrm{s}=1+\mathrm{k}^{-2}\left(\mathrm{EK}^{-1}-1\right), \\
\mathrm{E}=\int_{0}^{\pi / 2}\left(1-\mathrm{k}^{2} \mathrm{sin}^{2} \mathrm{v}\right)^{1 / 2} \mathrm{dv} \\
=\frac{\pi}{2} \mathrm{~F}\left(-\frac{1}{2}, \frac{1}{2} ; 1 ; \mathrm{k}^{2}\right)
\end{aligned}
$$

and $\mathrm{K}$ is given by Equation 15 .

Equation 20 has a form

$$
\left.\lambda \mathrm{u}_{1}=\lambda\left(1+\sigma_{2} \mu \mathrm{s}\right) \mathrm{u}+\text { (periodic terms in } \lambda \mathrm{u} \text { with the period } 4 \mathrm{~K}\right) \text {. }
$$

Therefore, by inversion, it follows that

$$
\lambda u=\lambda_{1} u_{1}+\left(\text { periodic terms in } \lambda_{1} u_{1}\right),
$$


where

$$
\lambda_{1}=\lambda\left(1+\sigma_{2} \mu \mathbf{s}\right)^{-1}
$$

Thus

$$
\begin{aligned}
\lambda \mathbf{u}=\nu \tau & -\sigma_{1} \sqrt{\mu} \mathrm{k}^{-1} \sin ^{-1}(\mathrm{k} \operatorname{sn} \nu \tau) \\
& +\sigma_{1}^{2} \mu \mathrm{cn} \nu \tau \mathrm{k}^{-1} \sin ^{-1}(\mathrm{ksn} \nu \tau)-\sigma_{2} \mu \mathrm{k}^{-2} \mathrm{Z}(\nu \tau)
\end{aligned}
$$

where

$$
\begin{aligned}
\nu & =2 \alpha_{1} \sqrt{\mu} \lambda_{1} \\
& =2 \alpha_{1}^{1 / 2} \mathrm{j}^{1 / 2} \Gamma^{1 / 2}(\alpha-\beta)^{1 / 2}\left(1-\sigma_{2} \mu \mathrm{s}\right),
\end{aligned}
$$

and $\mathrm{k}^{2}=(1-\beta)(\alpha-\beta)^{-1}$. The other notations, $\sigma_{1}$ and $\sigma_{2}$, are given by Equations 19; s by Equation 21 ; and $\alpha, \beta$ by Equations 16 . Then,

$$
\begin{aligned}
& x=\cos ^{2} \omega=\frac{\operatorname{sn}^{2}(\lambda u, k)}{a-\beta \operatorname{cn}^{2}(\lambda u, k)} \\
& x=\sqrt{\frac{\mathrm{j} \Gamma}{\alpha_{2}}}\left\{\left[1-\frac{\mathrm{j} \Gamma}{\beta_{0}}\left(\frac{1}{4} \frac{\beta_{1}^{2}}{\alpha_{2} \beta_{0}}-\frac{\gamma_{0}}{\beta_{0}}\right)\right] \operatorname{cn} \lambda \mathrm{u}-\frac{1}{2} \frac{\alpha_{3}}{\alpha_{2}} \sqrt{\frac{\mathrm{j} \Gamma}{\alpha_{2}}} \mathrm{cn}^{2} \lambda \mathrm{u}\right. \\
& \left.+\frac{\mathrm{j} \Gamma}{\beta_{0}}\left(\frac{1}{4} \frac{\beta_{1}^{2}}{\alpha_{2} \beta_{0}}-\frac{\gamma_{0}}{\beta_{0}}-\frac{1}{2} \frac{a_{4} \beta_{0}}{a_{2}^{2}}+\frac{5}{8} \frac{a_{3}^{2} \beta_{0}}{\alpha_{2}^{2}}\right) \mathrm{cn}^{3} \lambda \mathrm{u}\right\} \text {. }
\end{aligned}
$$

Therefore, we have, neglecting higher orders, the following forms as the solutions of Equation 1,

$$
\begin{aligned}
\cos \omega= & \left(\frac{\Gamma}{\beta_{0}}\right)^{1 / 2}\left[1+\frac{\mathrm{j} \Gamma}{2 \beta_{0}}\left(\frac{1}{2} \frac{\beta_{1}^{2}}{\alpha_{2} \beta_{0}}-\frac{2 \gamma_{0}}{\beta_{0}}-\frac{\beta_{2}}{\alpha_{2}}+\frac{3}{2} \frac{\alpha_{3} \beta_{1}}{\alpha_{2}^{2}}\right)\right] \mathrm{sn} \lambda \mathrm{u} \\
& -\left(\frac{\Gamma}{\beta_{0}}\right)^{1 / 2} \frac{\mathrm{j} \Gamma}{2 \beta_{0}}\left(\frac{1}{4} \frac{\beta_{1}^{2}}{\alpha_{2} \beta_{0}}-\frac{\gamma_{0}}{\beta_{0}}-\frac{\beta_{2}}{\alpha_{2}}+\frac{3}{2} \frac{\alpha_{3} \beta_{1}}{\alpha_{2}^{2}}\right) \mathrm{sn}^{3} \lambda \mathrm{u}, \\
\rho=\sqrt{\frac{\mathrm{j} \Gamma}{\alpha_{2}}} & {\left[1-\frac{\mathrm{j} \Gamma}{\beta_{0}}\left(\frac{1}{4} \frac{\beta_{1}^{2}}{\alpha_{2} \beta_{0}}-\frac{\gamma_{0}}{\beta_{0}}\right)\right] \operatorname{cn} \lambda \mathrm{u}-\frac{1}{2} \frac{\alpha_{3} \Gamma}{\alpha_{2}^{2}} \mathrm{j} \mathrm{cn}^{2} \lambda \mathrm{u} } \\
& -\frac{1}{2} \frac{\beta_{1} \Gamma}{\alpha_{2} \beta_{0}} \mathrm{j} \mathrm{sn}^{2} \lambda \mathrm{u}+\sqrt{\frac{\mathrm{j} \Gamma}{\alpha_{2}}} \frac{\mathrm{j} \Gamma}{\beta_{0}}\left(\frac{1}{4} \frac{\beta_{1}^{2}}{\alpha_{2} \beta_{0}}-\frac{\gamma_{0}}{\beta_{0}}\right. \\
& \left.-\frac{1}{2} \frac{\alpha_{4} \beta_{0}}{\alpha_{2}^{2}}+\frac{5}{8} \frac{\alpha_{3}^{2} \beta_{0}}{\alpha_{2}^{2} \alpha_{2}}\right) \mathrm{cn}^{3} \lambda \mathrm{u},
\end{aligned}
$$


where $\lambda_{\mathrm{u}}$ is given by Equation 22. The order of magnitude adopted here corresponds to the second order of Equation 7.

\section{Case ic: Revolution}

In this case a formula similar to the aforementioned one can be given:

$$
\mathrm{du}\left(1+\sigma_{1} \mu^{1 / 2} \mathrm{dn} \lambda \mathrm{u}+\sigma_{2} \mu \mathrm{dn}^{2} \lambda \mathrm{u}\right)=\mathrm{du}_{1}
$$

from which we have

$$
\begin{aligned}
\lambda \mathrm{u}_{1}= & \lambda \mathrm{u}+\sigma_{1} \mu^{1 / 2} \mathrm{am} \lambda \mathrm{u}+\sigma_{2} \mu \mathrm{E}(\lambda \mathrm{u}) \\
= & \left(1+\sigma_{1} \mu^{1 / 2} \frac{\pi}{2} \mathrm{~K}^{-1}+\sigma_{2} \mu \mathrm{EK}^{-1}\right) \lambda \mathrm{u} \\
& +\sigma_{1} \mu^{1 / 2}\left(\operatorname{am} \lambda \mathbf{u}-\frac{\pi}{2 \mathrm{~K}} \lambda \mathrm{u}\right)+\sigma_{2} \mu \mathrm{k}^{-2} Z(\lambda \mathrm{u}) .
\end{aligned}
$$

\section{Putting}

$$
\nu=2 \alpha_{2} \mu^{1 / 2}(1-\beta)^{1 / 2}\left(1+\sigma_{1} \mu^{1 / 2} \cdot \frac{\pi}{2} \mathrm{~K}^{-1}+\sigma_{2} \mu \mathbf{E K}^{-1}\right)-1
$$

we have, by inversion,

$$
\begin{aligned}
\lambda \mathrm{u}=\nu \tau & -\sigma_{1} \mu^{1 / 2}\left(\operatorname{am} \nu \tau-\frac{\pi}{2} \mathrm{~K}^{-1} \nu \tau\right) \\
& +\sigma_{1}^{2} \mu\left(\operatorname{dn} \nu \tau-\frac{\pi}{2} \mathrm{~K}^{-1}\right)\left(\operatorname{am} \nu \tau-\frac{\pi}{2} \mathrm{~K}^{-1} \nu \tau\right)-\sigma_{2} \mu \mathrm{Z}(\nu \tau),
\end{aligned}
$$

where

$$
\mathrm{k}^{2}=(\alpha-\beta)(1-\beta)^{-1} \text {. }
$$

We note that $\sigma_{1} \mu^{1 / 2}$ am $\lambda \mathrm{u}$, the second term of the right side of Equation 23, also contributes to the change of the period-contrary to Case $i b$, where the corresponding term, $\sigma_{1} \mu^{1 / 2} \mathrm{k}^{-1} \sin ^{-1}\left(\mathrm{k} \operatorname{sn} \lambda_{u}\right)$, is purely periodic provided $0 \leq \mathrm{k}^{2} \leq 1$. It is easily seen that, roughly speaking, Case $c$ corresponds to Case $b$ where $\mathrm{k}>1$. At any rate, for the former case we have the following solutions of Equation 1:

$$
\cos \omega=\sin \omega_{1}=\operatorname{sn} \lambda u\left[1+\frac{1}{2} \mathrm{j}\left(\frac{1}{4} \frac{\beta_{1}^{2}}{\alpha_{2} \beta_{0}}-\frac{\gamma_{0}}{\beta_{0}}-\frac{\beta_{2}}{\alpha_{2}}+\frac{3}{2} \frac{\alpha_{3} \beta_{1}}{\alpha_{2}^{2}}\right) \operatorname{cn}^{2} \lambda u\right] ;
$$




$$
\begin{aligned}
\rho=\rho+x= & \sqrt{\frac{\mathrm{j} \Gamma}{\alpha_{2}}} \mathrm{dn} \lambda \mathrm{u}-\frac{1}{2} \mathrm{j} \frac{\beta_{1}}{\alpha_{2}} \operatorname{sn}^{2} \lambda \mathrm{u}-\frac{1}{2} \mathrm{j} \frac{\Gamma \alpha_{3}}{\alpha_{2}^{2}} \mathrm{dn}^{2} \lambda \mathrm{u} \\
& -\mathrm{j} \sqrt{\frac{\mathrm{j} \Gamma}{\alpha_{2}}}\left(\frac{1}{4} \frac{\beta_{1}{ }^{2}}{\alpha_{2} \beta_{0}}-\frac{\gamma_{0}}{\beta_{0}}\right) \operatorname{sn}^{2} \lambda \mathrm{u} \operatorname{dn} \lambda \mathrm{u}+\left(\frac{\mathrm{j} \Gamma}{\alpha_{2}}\right)^{3 / 2}\left(-\frac{1}{2} \frac{\alpha_{4}}{\alpha_{2}}+\frac{5}{8} \frac{\alpha_{3}^{2}}{\alpha_{2}^{2}}\right) \mathrm{dn}^{3} \lambda \mathrm{u} .
\end{aligned}
$$

\section{GENERAL SCHEME OF THE FURTHER APPROXIMATIONS}

In order to solve the equations with higher order terms, it is first necessary to solve an equation such as Equation 6 which contains these terms. Even if the original equations of motion are limited to finite terms, the Equation 6-type of equation is, in general, an algebraic equation with $\cos ^{2} \omega$ as the unknown. When combined with the second equation of Equation 4 , we find that $(d / d \tau)\left(\cos ^{2} \omega\right)$ is equal to an algebraic function in $\cos ^{2} \omega$. The solution of a differential equation of this kind would involve Abelian integrals-many valued functions, more complicated than the elliptic integrals. Therefore, the solution becomes so complicated that we cannot see the general character of the motion at a first glance. However, if we restrict ourselves to the solution of a real function, the complicacy would be much reduced.

In this section we are concerned with the cases where the character of motion is quite similar to the former cases, except for slight changes caused by the higher order terms.

Now, we consider the equations of motion in the form:

$$
\begin{aligned}
& \frac{\mathrm{d} \chi}{\mathrm{d} \tau}=-\frac{\partial \Psi}{\partial \omega_{1}}, \\
& \frac{\mathrm{d} \omega_{1}}{\mathrm{~d} \tau}=\frac{\partial \Psi}{\partial \chi},
\end{aligned}
$$

where

$$
\Psi=j a_{0}\left(\sin ^{2} \omega_{1}, j\right)+\sum_{2} a_{n}\left(\sin ^{2} \omega_{1}, j\right) x^{n}
$$

and $a_{n}(n=2,3, \cdots)$ is a power series in $j, a_{2}\left(\sin ^{2} \omega_{1}, 0\right)=a_{2,0}$ and is a constant which corresponds to the previous case if $a_{2,0}=a_{2}$.

From the integral $\Psi-\mathrm{j} \Gamma=0$, we obtain

$$
4 a_{2}^{2} \chi^{2}\left(1+\sum a_{n}^{*} \chi^{n-2}\right)=4 a_{2} j\left(\Gamma-a_{0}\right) \equiv \eta^{2}
$$

from which

$$
2 \mathbf{a}_{2} x=\eta+\sum_{1} b_{n} \eta^{n}
$$


Thus, we have

$$
\left(\frac{\partial \Psi}{\partial \chi}\right)^{-1} \mathrm{~d} \omega_{1}=\mathrm{d} \tau
$$

or

$$
\eta^{-1}\left(1+\sum \mathbf{c}_{\mathrm{n}} \eta^{\mathrm{n}}\right)^{-1} \mathrm{~d} \omega_{1}=\text { constant } \cdot \mathrm{d} \tau
$$

If the solution of $\eta^{-1} \mathrm{~d} \omega_{1}=\mathrm{du} *$ could be obtained in a simple way, as in the previous cases, then using $u^{*}$ as an intermediary parameter would be a direct extension of the previous cases. But this integral is, in general, an Abelian integral as we have just stated. A general treatment is not suitable for practical purposes. As an alternative, we shall consider the following situations.

\section{a: Case of moderate eccentricity}

Let $a_{0}\left(\sin ^{2} \omega_{1}, 0\right)=a_{0,1} \sin ^{2} \omega_{1}, a_{0,1}>0$; then the solution of $a_{0}\left(\sin ^{2} \omega_{1}, 0\right)-\Gamma=0$ is

$$
\sin ^{2} \omega_{1}=\left(a_{0,1}\right)^{-1} \Gamma
$$

Now, we denote one of the solutions by $a^{-1}$ :

$$
a_{0}\left(\sin \omega_{1}, j\right)-\Gamma=0,
$$

the first approximation of which is Equation 24. We then have

$$
4 a_{2} j\left[\Gamma-a_{0}\left(\sin ^{2} \omega_{1}, j\right)\right]=\mu_{1}\left(1-\alpha \sin ^{2} \omega_{1}\right)\left(1+\sum A_{n} \sin ^{2 n} \omega_{1}\right),
$$

where $\mu_{1}=4 \Gamma a_{2,0} j$. Therefore,

$$
\eta=\sqrt{\mu_{1}} \xi\left(1+\sum \beta_{n} \sin ^{2 n} \omega_{1}\right)
$$

where $\xi^{2}=1-\alpha \sin ^{2} \omega_{1}$ and

$$
\xi^{-1}\left[1+\sum \mu_{1}^{n / 2} f_{n}\left(\sin ^{2} \omega_{1}\right) \xi^{n}\right]^{-1} d \omega_{1}=\mu_{1}^{1 / 2} d \tau
$$

Now

$$
\xi^{-1} \mathrm{~d} \omega_{1}=\mathrm{du}
$$

Here we again divide the problem into two subcases:

Case ib: $a>1$

We have

$$
\sqrt{a} \sin \omega_{1}=\operatorname{sn}\left(\lambda u, a^{-1 / 2}\right),
$$




$$
\begin{aligned}
\xi & =\sqrt{1-\alpha \sin ^{2} \omega_{1}} \\
& =\operatorname{cn}\left(\lambda u, \alpha^{-1 / 2}\right), \\
\lambda & =\alpha^{1 / 2} ;
\end{aligned}
$$

then

$$
\left[1+\sum \mu^{\mathrm{n} / 2} \mathrm{f}_{\mathrm{n}}\left(\alpha^{-1} \operatorname{sn}^{2} \alpha^{1 / 2} \mathrm{u}\right)\left(\mathrm{cn}^{\mathrm{n}} \alpha^{1 / 2} \mathrm{u}\right)\right]^{-1} \mathrm{du}=\mu_{1}^{1 / 2} \mathrm{~d} \tau
$$

or

$$
\left[1+\sum \mu^{\mathrm{n} / 2} \mathrm{~g}_{\mathrm{n}}\left(\alpha^{-1} \mathrm{sn}^{2} \alpha^{1 / 2} \mathrm{u}\right) \mathrm{cn}^{\mathrm{n}} \alpha^{1 / 2} \mathrm{u}\right] \mathrm{du}=\mu_{1}^{1 / 2} \mathrm{~d} \tau
$$

where $g_{n}$ is a power series in $j$ with the coefficients polynomials in $a^{-1} \operatorname{sn}^{2} \lambda u$. Therefore, the integration of Equation 26 is expressed as

$$
\mathrm{u}+\sum \mathrm{B}_{\mathrm{n}} \int \mathrm{cn}^{\mathrm{n}} \lambda \mathrm{u} \mathrm{du}=\mu_{1}^{1 / 2} \tau
$$

where $\mathrm{B}_{\mathrm{n}}$ is a numerical constant. This integration is carried out by the use of the Jacobian elliptic function and the Jacobian zeta-function (see Reference 10, p. 164). Thus, the final form is expressed by

$$
\lambda \mathrm{u}+\text { a periodic function of } \lambda \mathrm{u}=\text { constant } \cdot \tau \text {, }
$$

where the constant factor in the right side is $\mu_{1}^{1 / 2} \lambda[1+O(j)]$. This is an extension of Equation 20 .

Case ic: $a<1$

From Equation 25 we have

$$
\begin{gathered}
\sin \omega_{1}=\operatorname{sn}\left(\mathrm{u}, \alpha^{1 / 2}\right) \\
\xi=\sqrt{1-\alpha \sin ^{2} \omega_{1}}=\operatorname{dn}\left(\mathrm{u}, \alpha^{1 / 2}\right) ;
\end{gathered}
$$

therefore,

$$
\left[1+\Sigma \mu^{\mathrm{n} / 2} \mathrm{f}_{\mathrm{n}}\left(\operatorname{sn}^{2} \mathrm{u}\right) \mathrm{dn}^{\mathrm{n}} \mathrm{u}\right]^{-1} \mathrm{du}=\mu_{1}^{1 / 2} \mathrm{~d} \tau
$$


or

$$
\left[1+\sum \mu^{n / 2} g_{n}\left(s^{2} u\right) d n^{n} u\right] \cdot d u=\mu_{1}^{1 / 2} d \tau
$$

In this case we have, instead of Equation 27,

$$
u+\Sigma C_{n} \int d n^{n} u d u=\sqrt{l_{1}} \tau
$$

where the integration will be carried out, as above, by using the Jacobian elliptic function and the Jacobian zeta-function. Thus we have

$$
\mathrm{u}+\text { a periodic function of } \mathrm{u}=\text { constant } \cdot \tau \text {, }
$$

where the constant factor in the right side again is $\mu_{1}^{1 / 2}[1+O(j)]$.

\section{$\beta:$ Case of small eccentricity}

In this case the most important factor in $\Gamma-a_{0}\left(\sin ^{2} \omega_{1}, j\right)$ is not the $1-\alpha \sin ^{2} \omega_{1}$ term, as in the previous case, but is $\left(1-\alpha \sin ^{2} \omega_{1}\right)\left(1-\beta \sin ^{2} \omega\right)$, where both $\alpha$ and $\beta$ are of the order of 1 . Therefore we cannot expand each of the above factors into power series beginning with a constant term.

Or more concretely, hereafter $a_{0,1}$ is assumed to be of the order of $j: a_{01}=j a_{0,1}^{\prime}$ and, also, it is assumed that

$$
\lim _{j \rightarrow 0} j^{-1} a_{0}\left(\sin ^{2} \omega_{1}, j\right)=a_{0,1}^{\prime} \sin ^{2} \omega_{1}+a_{0,2} \sin ^{4} \omega_{1}
$$

where $a_{0,1}^{\prime}$ corresponds to $\beta_{0}^{\prime}$, and $a_{0,2}$ to $-(1 / 4) \beta_{1}^{2} \alpha_{2}^{-1}$. Also, we denote by $\alpha^{-1}, \beta^{-1}$ the solutions of

$$
a_{0}\left(\sin ^{2} \omega_{1}, j\right)-j \Gamma^{\prime}=0
$$

which approach the solutions of

$$
\Gamma^{\prime}-a_{0,1}^{\prime} \sin ^{2} \omega_{1}-a_{0,2} \sin ^{4} \omega_{1}=0 .
$$


As before, we have

$$
4 a_{2} j\left[j \Gamma^{\prime}-a_{0}\left(\sin ^{2} \omega_{1}, j\right)\right]=\mu_{1}\left(1-\alpha \sin \omega_{1}\right)\left(1-\beta \sin ^{2} \omega_{1}\right)\left(1+\sum A_{n} \sin ^{2 n} \omega_{1}\right),
$$

where $\mu_{1}=4 \Gamma^{\prime} a_{2,0} j^{2}$. Therefore,

$$
\begin{aligned}
\eta & =\mu_{1}^{1 / 2} \chi_{0}\left(1+\sum \beta_{n} \sin ^{2 n} \omega_{1}\right) \\
x_{0}^{2} & =\left(1-\alpha \sin ^{2} \omega_{1}\right)\left(1-\beta \sin ^{2} \omega_{1}\right)
\end{aligned}
$$

and, as before,

$$
\chi_{0}^{-1}\left[1+\sum \mu_{1}^{n / 2} f_{n}\left(\sin ^{2} \omega_{1}\right) \chi_{0}^{n}\right]^{-1} d \omega_{1}=\mu_{1}^{1 / 2} d \tau
$$

Since $\chi_{0}^{-1} \mathrm{~d} \omega_{1}=\mathrm{du}$, we have, for case $i$ (except for the critical cases):

$$
x=\sin ^{2} \omega_{1}=\frac{\operatorname{sn}^{2}(\lambda u, k)}{p-q \operatorname{cn}^{2}\left(\lambda u_{1} k\right)}
$$

where $\lambda, \mathrm{k}, \mathrm{p}$, and $\mathrm{q}$ can be obtained in Table 1 , according to the respective cases. Further,

$$
x_{0}=\frac{(p-q) \mathrm{cn}^{\epsilon} \lambda \mathrm{udn}^{\epsilon} \epsilon_{2 \mathrm{u}}}{\mathrm{p}-\mathrm{q} \mathrm{cn}^{2}\left(\lambda \mathbf{u}_{1} \mathrm{k}\right)}
$$

where $\epsilon_{1}, \epsilon_{2}=0$ or 1 , but both are not zero at the same time.

Now, from Equation 28 we have

$$
\left[1+\sum \mu_{1}^{\mathrm{n} / 2} \mathrm{f}_{\mathrm{n}}(\mathrm{x}) \chi_{0}^{\mathrm{n}}\right]^{-1} \mathrm{du}=\mu_{1}^{1 / 2} \mathrm{~d} \tau
$$

or

$$
\left[1+\sum \mu_{1}^{n / 2} g_{n}(x) \chi_{0}^{n}\right] \quad d u=\mu_{1}^{1 / 2} d \tau
$$

where $g_{n}$ is a polynomial of $x$. Thus, for $n$ even, $g_{n}$ has the form

$$
\mathrm{g}_{\mathrm{n}}=\mathrm{G}_{1}\left(\mathrm{sn}^{2} \lambda \mathbf{u}\right)
$$

where $G_{1}$ denotes a rational function of the argument. The integration of Equation 32 produces an elliptic integral of the third kind, in general, unlike Case a (Reference 10, p. 164). On the other hand, 
for $\mathrm{n}$ odd,

$$
\mathrm{g}_{\mathrm{n}}=\mathrm{G}_{2}\left(\mathrm{sn}^{2} \lambda \mathrm{u}\right) \mathrm{cn}^{\epsilon_{1}} \lambda \mathrm{u} \mathrm{dn}{ }^{\epsilon_{1}} \lambda \mathrm{u}
$$

the integration of which requires only the Jacobian elliptic functions.

For Case $i$, however, the equations corresponding to Equations 29 and 30 have the different forms; but all the functions $\mathrm{g}_{\mathrm{n}}$ have a single form such as Equation 32.

In any case, we have, after integration of Equations 28 or 32, the form

$$
\lambda \mathrm{u}+\text { a periodic function of } \lambda \mathrm{u}=\text { constant } \cdot \tau,
$$

from which we obtain

$$
\lambda \mathrm{u}=\text { constant } \cdot \tau+\text { a periodic function of constant } \cdot \tau \text {. }
$$

It should be noted that the odd power terms in Equation 31 do not contribute any change to the period of libration, where $\epsilon_{1}=0$, which corresponds to the case of libration.

\section{DISCUSSION}

The denominator of the right side of Equation 11 is a square root of a quartic of $\mathbf{x}$. A general treatment of such a case was carried out by Andoyer (Reference 11) and extended by Hagihara (Reference 12) in connection with the libration problem of asteroids (see also Reference 13). Both of them used the $\zeta$-function of Weierstrass; nevertheless, we feel the integration form described here is more suitable for this special case, owing to the fact that only real functions are involved. For example, if $\beta \rightarrow 0$ in Equation 10,

$$
\frac{\mathrm{d} \omega_{1}}{\sqrt{1-\mathrm{k}^{2} \sin ^{2} \omega_{1}}}=\mathrm{du},
$$

where $\mathrm{k}^{2}=\alpha$, from which, if $0<\alpha<1$, we have $\sin \omega_{1}=\operatorname{sn} u$, which is, of course, a limiting case of $i c$, etc. Thus the present work represents some natural extensions of the derivation of well-known Jacobian elliptic functions.

For the numerical computation, integrating Equation 17 in the literal form may not necessarily be the best way, for a direct numerical integration of Equation 17 is more effective, since its denominator generally does not become very small-otherwise, the numerical integration would lose its validity. However, the problem of inversion of a numerical function still would remain. 


\section{CONCLUSION}

Equations 1, which have terms up to second order of $j$, have been solved both for the case of small eccentricity and for moderate eccentricity. In the former, the intermediary solutions themselves are sufficient for the present discussion, since the solutions have an accuracy of the order of 1 for $\cos \omega$ (neglecting the order of $j$ ) and of the order of $j$ for $\rho$ (neglecting $j^{2}$ ). This accuracy corresponds to the order of $j^{2}$ in the Hamiltonian, since $\rho$ has the factor of $j$ and the argument of the solution is neither $\tau$ alone nor multiplied by a constant factor of order of 1 , but $\tau$ multiplied by that of order $j$.

On the other hand, in the second or normal cases, the solutions have the accuracy of order $j$ for $\cos \omega$ and $\mathrm{j}^{3 / 2}$ for $\rho$, which corresponds to $\mathrm{j}^{2}$ in the Hamiltonian.

\section{ACKNOWLEDGMENTS}

The author would like to express his gratitude to Mr. I. G. Izsak and to Drs. P. Musen and Y. Kozai for their discussions.

\section{REFERENCES}

1. Hori, G. I., "The Motion of an Artificial Satellite in the Vicinity of the Critical Inclination," Astronom. J. 65(5):291-300, June 1960.

2. Garfinkel, B., "On the Motion of a Satellite in the Vicinity of the Critical Inclination," Astronom. J. 65(10):624-627, December 1960.

3. Hagihara, Y., "Libration of an Earth Satellite With Critical Inclination," Smithsonian Contrib. to Astrophys. 5(5):39-51, 1961.

4. Kozai, Y., "Motion of a Particle With Critical Inclination in the Gravitational Field of a Spheroid," Smithsonian Contrib. to Astrophysics 5(5):53-58, 1961.

5. Izsak, I. G., "On the Critical Inclination in Satellite Theory," Smithsonian Inst., Astrophys. Observ., Res. in Space Sci., Spec. Rept. No. 90, March 14, 1962.

6. Neville, E. H., "Jacobian Elliptic Functions," 2nd Ed., Oxford: Clarendon Press, 1951.

7. Whittaker, E. T., and Watson, G. N., "A Course of Modern Analysis," 4th Ed., Cambridge: University Press, 1927.

8. Kozai, Y., "Second-Order Solution of Artificial Satellite Theory without Air Drag"" Astronom. J. 67(7):446-461, September 1962.

9. Hancock, H., "Elliptic Integrals," New York: Dover, 1958. 
10. Gröbner, W., and Hofreiter, N., "Integraltafel. Vol. 1, Unbestimmte Integrale," 2nd Ed., Vienna: Springer-Verlag, 1957.

11. Andoyer, H., "Contribution à la Théorie des Petites Planètes dont le Moyen Mouvement est Sensiblement Double de celui de Jupiter," Bulletin Astronomique 20:321-356, September 1903.

12. Hagihara, Y., "On the General Theory of Libration," Japan. J. Astron. and Geophys. 21(1-2):2943, 1944.

13. Halphen, G. H., "Traité des Fonctions Elliptiques et de leurs Applications," Paris: GauthierVillars, 1886, Vol. 1, Chapters 4 and 5. 
Page Intentionally Left Blank 


\section{Appendix A}

\section{List of Symbols}

$c_{d}, c_{n}, d_{n}$

$e_{f}, e_{g}, e_{h}$

E

$f_{j}, g_{j}, h_{j}$

$\mathrm{J}_{\mathrm{n}}$

k

$\mathrm{K}$

$\wp$

$s_{n}$

$t$

$\mathrm{T}$

u

$x=\sin ^{2} \omega_{1}=\cos ^{2} \omega$

$\alpha, \beta, \alpha_{i}, \beta_{i}, \gamma_{i}$

$\Gamma=\mathbf{j} \Gamma^{\prime}$

$\lambda$

$\rho$

$\omega$

$\omega_{1}=\pi / 2-\omega$ $\omega_{f}, \omega_{g}, \omega_{h}$
Jacobian elliptic functions (see pages 12, 8, and 9)

Parameter of Weierstrassian elliptic function (see page 6)

Complete elliptic integral of the second order (see page 17)

First order elliptic function associated with the $\wp$-function (see Reference 6 . and page 7)

Harmonic coefficient of the earth's potential (see page 2)

Modulus of the Jacobian elliptic function (see pages 7 and 11)

Complete elliptic integral of the first order (see page 14)

Weierstrassian elliptic function (see page 6)

Jacobian elliptic function (see page 8 )

Time

Period of the solutions (see page 14)

$u$ will equal $t$, except for the constant factor, if higher orders are neglected (see page 13)

\section{Constants}

Energy constant (see page 4)

Constant factor in the argument of the Jacobian elliptic functions (see page 12)

Variable related to the eccentricity (see Reference 5 and page 2)

Mean argument of the perigee

Semi-periods associated with the Weierstrassian elliptic function (see page 7) 